\title{
Synthesis and Characterization of 1,3,4-Oxadiazoles Derivatives from 1,2,3,4-Tetrahydroisoquinoline and their Bioevalution (Antibacterial \& Antifungal)
}

\author{
N. KRISHNA RAO ${ }^{1}$, M. SURENDRA BABU ${ }^{2 *}$, M. V. BASAVESWARA RAO $^{1}$, \\ R. KESHAVI ${ }^{3}$, N. SUNDARA RAO ${ }^{3}$, Y. L. N. MURTHY ${ }^{3}$ and SHAIK LAKSHMAN ${ }^{4}$ \\ ${ }^{1}$ Chemistry Department, Krishna University, Machilipatnam, India \\ ${ }^{2}$ Department of Chemistry, GITAM University, Hyderabad, India \\ ${ }^{3}$ Department of Organic Chemistry, Andhra University, Visakhapatnam, India \\ ${ }^{4}$ Department of Chemistry, GITAM University, Visakhapatnam, India \\ surendra@gitam.edu
}

Received 7 December 2016 / Revised 1 March 2017 /Accepted 28 March 2017

\begin{abstract}
New derivatives of 1,3,4-oxadiazole, synthesized by the condensation of different substituted benzoic acids with 2-(1-phenyl-3,4-dihydroisoquinoline-2(1H)-yl) acetohydrazide using $\mathrm{SOCl}_{2}$ as well as $\mathrm{POCl}_{3}$ as cyclization reagents. Ethyl-2-(1-phenyl-1,2,3,4-tetrahydroisoquinoline)ethaonate was synthesized from 1,2,3,4-Tetrahydroisoquinoline and it was converted to the 2-(1phenyl-3,4-dihydroisoquinoline-2(1H)-yl)acetohydrazide. This compound is the key intermediate for the 1,3,4-oxadiazole compounds. All the synthesized molecules were characterized by advanced spectral data and also studied their biological activity.
\end{abstract}

Keywords: 1,2,3,4-Tetrahydroisoquinoline, Ethyl-2-(1-phenyl-3,4-dihydro isoquinoline-2(1H)-yl) acetate, 2-(1-Phenyl-3,4-dihydroisoqunoline-2(1H)-yl)acetohydrazide, 1,3,4-Oxadiazole, Cyclization reagents and bioevolution

\section{Introduction}

1,3,4-Oxadiazole is a five membered heterocyclic compound which possess two nitrogen atoms and one oxygen atom. This compound derived from furan by substitution of two methylene groups (-CH-) with two pyridine type nitrogen $(-\mathrm{N}=)^{1-2}$. Among three isomers of oxadiazoles such as 1,2,4-oxadiazole, 1,2,3-oxadiazole and 1,3,4-oxadiazole, the most important compound is 1,3,4-oxadiazole.

1,3,4-Oxadiazole was found to possess a wide spectrum of pharmacological activity such as antibacterial ${ }^{3-4}$, anti fungal ${ }^{5-6}$, anthelminitic ${ }^{7}$, anti tubercular ${ }^{8}$, anti-infective ${ }^{9}$, anti cancer ${ }^{10}$, anti-HIV $^{11}$, anti oxidant ${ }^{12}$, analgesic ${ }^{13-14}$, anti histanamic ${ }^{15}$, insectivdal ${ }^{16}$, anti-inflammatory ${ }^{17}$, and anti convulsant ${ }^{18-19}$. 
1,3,4-Oxadiazoles played a crucial role in the development of new heterocyclic's and also used extensively in organic synthesis ${ }^{20}$. In this present investigations, we report the synthesis of 1,3,4-oxadiazole derivatives starting from 2-phenylethylamine and benzoic acid. The synthesis is described in Scheme 1.
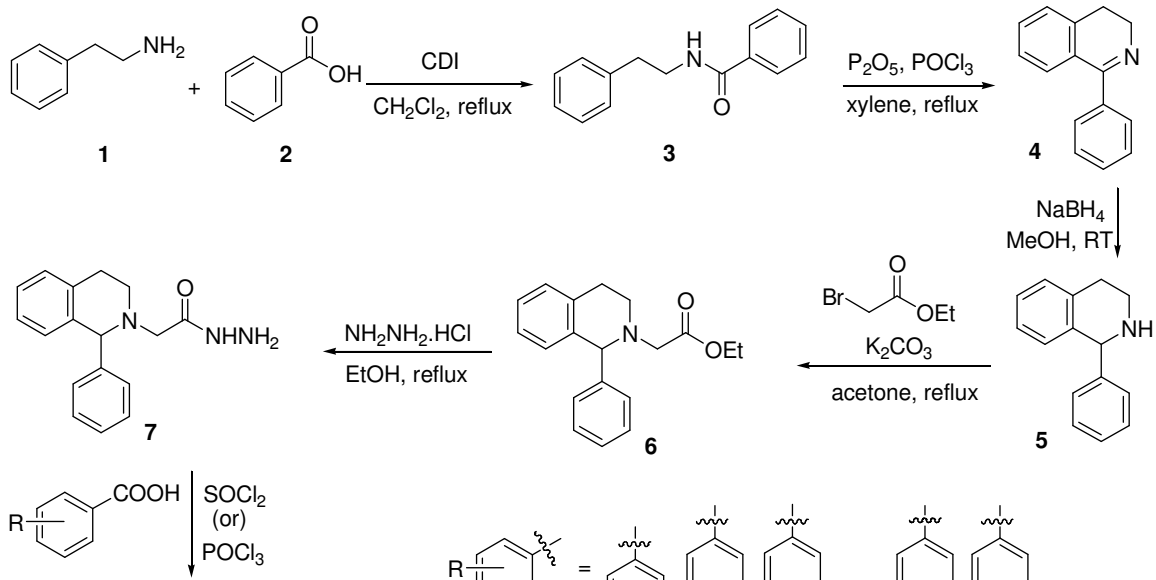<smiles>[R]c1cccc(-c2nnc(CN3CCc4ccccc4C3c3ccccc3)o2)c1</smiles>

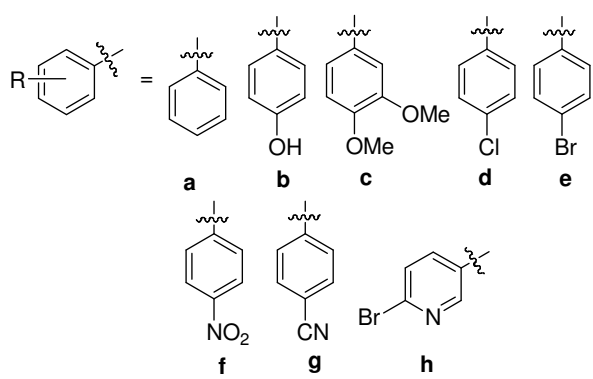

Scheme 1. Synthesis of 1,3,4-oxadiazoles derivatives

\section{Experimental}

Melting points were determined by open capillary method and were uncorrected. ${ }^{1} \mathrm{H}$ NMR and ${ }^{13} \mathrm{C}$ NMR spectra were recorded on a Bruker-FT-NMR-400 $\mathrm{MHz}$ spectrophotometer using $\mathrm{CDCl}_{3}$ as solvent and TMS as an internal standard. LC-Mass spectra were recorded on a Varian Atlas CH-7 mass spectrometer.

\section{Experimental procedure and Characterization}

Synthesis of N-(2-phenyl ethyl)-benzamide (3)

A mixture of 2-phenylamine (1,1 equi.) and benzoicacid (2,1.2 equi.) was mixed in dichloro methane (DCM) followed by 1'-carbonyldiimidazole (CDI) were introduced into a $250 \mathrm{~mL}$ R.B. flask. The reagents were heated and stirred under reflux condition. The reaction was monitored by TLC and after completion of the reaction, the reaction mixture was washed with saturated brine solution, dried over anhydrous $\mathrm{Na}_{2} \mathrm{SO}_{4}$ and concentrated to get crude product (3). The yield of the product obtained was $95 \%$ after column chromatography (silica gel; solvent system -hexane and ethyl acetate 70:30).

${ }^{1} \mathrm{H}$ NMR $\left(400 \mathrm{MHz}, \mathrm{CDCl}_{3}\right) \delta: 7.70(\mathrm{~d}, J=8.4 \mathrm{~Hz}, 2 \mathrm{H}), 7.49-7.30(\mathrm{~m}, 5 \mathrm{H}), 7.25-7.22$ $(\mathrm{m}, 3 \mathrm{H}), 6.32(\mathrm{br} \mathrm{s}, 1 \mathrm{H}), 3.68-3.73(\mathrm{~m}, 2 \mathrm{H}), 2.93(\mathrm{t}, J=7.2 \mathrm{~Hz}, 2 \mathrm{H}) .{ }^{13} \mathrm{C}$ NMR $(100 \mathrm{MHz}$, $\left.\mathrm{CDCl}_{3}\right) \delta: 167.6,139.0,134.7,131.4,128.8,128.7,128.6,126.9,126.6,41.2$, 35.7. LCMS $(\mathrm{m} / \mathrm{z})$ : 226.2. Mol. formula: $\mathrm{C}_{15} \mathrm{H}_{15}$ NO. Elemental analysis: Calculated: C-79.97, H-6.71, N- 6.22. Obtained: C-79.98, H-6.69, N- 6.20. 


\section{Synthesis of 1-pheny-3,4-dihydroisoquinoline (4)}

Compound 3 was dissolved in xylene and $\mathrm{P}_{2} \mathrm{O}_{5}$ (1.2 equiv) followed by $\mathrm{POCl}_{3}$ (3.0 equiv) at room temperature. The reaction mixture was then refluxed and monitored by TCL. After completion of the reaction, the hot reaction solution was poured into ice and carefully the $\mathrm{pH}$ of solution adjusted to 12 using $20 \% \mathrm{NaOH}$. Later, water was added to dissolve precipitated phosphates. Then toluene was added to extract using $1 \mathrm{~N} \mathrm{HCl}$. The aqueous fraction was made alkaline using 20\% $\mathrm{NaOH}$ under ice-cooling condition. Again the product was extracted using toluene, dried over anhydrous $\mathrm{Na}_{2} \mathrm{SO}_{4}$. Solvent was evaporated under vacuum which yielded the desired product 4 in $92 \%$ as yellow colored viscous liquid.

${ }^{1} \mathrm{H}$ NMR (400 MHz, $\left.\mathrm{CDCl}_{3}\right)$ 8: 7.62-7.52 (m, 2H), 7.39-7.30 (m, 4H), 7.22-7.18 $(\mathrm{m}, 3 \mathrm{H}), 3.80(\mathrm{t}, \mathrm{J}=6.8 \mathrm{~Hz}, 2 \mathrm{H}), 2.74(\mathrm{t}, \mathrm{J}=6.4 \mathrm{~Hz}, 2 \mathrm{H}) .{ }^{13} \mathrm{C} \mathrm{NMR}\left(100 \mathrm{MHz}, \mathrm{CDCl}_{3}\right) \delta$ : 165.11, 141.18, 139.00, 138.69, 131.02, 130.50, 129.16, 129.04, 128.06, 127.19, 126.03, 45.84, 27.33. LCMS $(\mathrm{m} / \mathrm{z})$ : 206.18. Molecular formula: $\mathrm{C}_{15} \mathrm{H}_{13} \mathrm{~N}$. Elemental analysis Calculated: C-86.92, H-6.32, N-6.76, obtained: C-86.93, H-6.30, N-6.75.

Synthesis of 1-phenyl-1,2,3,4-tetrahydroisoquinoline (5)

1-Phenyl-3,4-dihydroisoquinoline dissolved in methanol (4, 1.0 equiv) was taken in RB flask, and then cooled to $0{ }^{\circ} \mathrm{C}$. Sodium borohydride (1.5 equiv.) was added in portions at $0{ }^{\circ} \mathrm{C}$ and left for few hours to attain room temperature. After 3 hours stirring, methanol was evaporated and the residue was taken in dichloromethane. Water was added in fractions and washed twice with dichloromethane. All the dichloromethane fractions were combined, dried over anhy. $\mathrm{Na}_{2} \mathrm{SO}_{4}$. Solvent was removed and the residue was purified by column chromatography using silica gel as stationary phase and mixture of ethyl acetate and hexanes as eluent to obtain pure racemic 1-phenyl-1,2,3,4-tetrahydroisoquinoline (5) in $88 \%$ yield as white solid.

${ }^{1} \mathrm{H}$ NMR $\left(400 \mathrm{MHz}, \mathrm{CDCl}_{3}\right)$ 8: 7.30-7.18 (m, 5H), 7.12-7.07 (m, 2H), 6.99-6.95 $(\mathrm{m}, 1 \mathrm{H}), 6.65(\mathrm{~d}, J=8.0 \mathrm{~Hz}, 1 \mathrm{H}), 5.02(\mathrm{~s}, 1 \mathrm{H}), 3.08-2.92(\mathrm{~m}, 3 \mathrm{H}), 2.75-2.84(\mathrm{~m}, 1 \mathrm{H}) .{ }^{13} \mathrm{C}$ NMR $\left(100 \mathrm{MHz}, \mathrm{CDCl}_{3}\right) \delta: 142.4,136.0,133.4,127.3,127.1,126.5,126.2,125.6,124.6$, 123.83, 59.9, 39.6, 27.0, LCMS $(\mathrm{m} / \mathrm{z}): 210.16$, Molecular formula: $\mathrm{C}_{15} \mathrm{H}_{15} \mathrm{~N}$. Elemental analysis; Calculated: C-86.08, H-7.22, N-6.69, obtained: C-86.10, H-7.21, N-6.68.

\section{Synthesis of ethyl, 2-(1-phenyl-3,4-dihydroisoqinoline-2(1H)-yl)acetate (6)}

1-Phenyl-1,2,3,4-tetrahydroisoquinoline was treated with ethyl bromoacetate in the presence of strong base $\left(\mathrm{K}_{2} \mathrm{CO}_{3}\right)$ in acetone. The mixture was stirred on magnetic stirrer under reflux for 3 hours. The reaction was monitored by TLC. After completion of the reaction, the crude was worked up with ethyl acetate and washed with anhydrous sodium carbonate. The resultant compound was purified by recrystallization in ethanol.

${ }^{1} \mathrm{H} \quad \mathrm{NMR} \quad\left(400 \quad \mathrm{MHZ}, \mathrm{CDCl}_{3}\right) \quad \delta: 7.3-7.25 \quad(\mathrm{~m}, 1 \mathrm{H}), \quad 7.12 \quad(\mathrm{~d}, \mathrm{~J}=6.0 \mathrm{~Hz}, 2 \mathrm{H}), \quad 7.08$ $(\mathrm{d}, \mathrm{J}=7.21 \mathrm{~Hz}, 1 \mathrm{H}), 6.99(\mathrm{~d}, \mathrm{~J}=7.6 \mathrm{~Hz}, 1 \mathrm{H}), 4.96(\mathrm{~s}, 1 \mathrm{H}), 4.15-4.13(\mathrm{~m}, 2 \mathrm{H}), 3.62-3.17(\mathrm{~m}$, $2 \mathrm{H}), 3.06-3.04(\mathrm{~m}, 2 \mathrm{H}), 2.86-2.82(\mathrm{~m}, 2 \mathrm{H}), 1.26-1.23(\mathrm{~m}, 3 \mathrm{H}) .{ }^{13} \mathrm{C} \mathrm{NMR}\left(100 \mathrm{MHz}, \mathrm{CDCl}_{3}\right)$ $\delta: 170.1,143.5,141.9,137.6,132.8,129.2,128.0,127.6,126.3,126.1,126.0,125.8,60.9$, 57.6, 52.4, 48.9, 27.1, 14.5. LCMS $(\mathrm{m} / \mathrm{z})$ : 295.22. Molecular formula: $\mathrm{C}_{19} \mathrm{H}_{21} \mathrm{NO}_{2}$. Elemental analysis; Calculated: C-77.26, H-7.17, N-4.74, O-10.83, obtained: C-77.28, H-7.16, N-4.73, O-10.81.

\section{Synthesis of 2-(1-phenyl-3,4-dihydroisoquinoline-2(1H)-yl)acetohydrazide (7)}

The compound 7 was synthesized by the mixture of ethyl-2-(1-phenyl-3,4-dihydroisoquinoline-2(1H)-yl) acetate, hydrazine hydrocholoride and ethanol. The reaction mixture 
subjected to reflux for $4 \mathrm{~h}$ on magnetic stirrer. After completion of the reaction, the crude was worked up with ethyl acetate and washed with anhydrous sodium carbonate. The resultant compound was purified by recrystallization in ethanol.

${ }^{1} \mathrm{H}$ NMR $\left(400 \mathrm{MHZ}, \mathrm{CDCl}_{3}\right) \delta: 8.10(\mathrm{~s}, 1 \mathrm{H}), 7.36-7.12(\mathrm{~m}, 10), 6.66(\mathrm{~d}, \mathrm{~J}=7.6 \mathrm{~Hz}, 1 \mathrm{H})$, $4.61(\mathrm{~s}, 1 \mathrm{H}), 3.34-3.09(\mathrm{~m}, 2 \mathrm{H}), 2.92-2.77(\mathrm{~m}, 4 \mathrm{H}) .{ }^{13} \mathrm{C} \mathrm{NMR}\left(100 \mathrm{MHz}, \mathrm{CDCl}_{3}\right)$ 8: 171.1, 142.7, 137.6, 133.7, 129.6, 128.8, 128.7, 128.5, 126.3, 126.0, 125.9, 68.9, 57.6, 49.9, 29.4. LCMS (m/z): 280.98, Molecular formula: $\mathrm{C}_{17} \mathrm{H}_{19} \mathrm{~N}_{3} \mathrm{O}$. Elemental analysis; Calculated: C72.57, H-6.81, N-614.94, O-5.39, obtained: C-72.59, H-6.79, N-14.92, O-5.38.

General procedure for synthesis of 1,3,4-oxadiazoles

2-(1-Phenyl-3,4-dihydroisoquinoline-2(1H)-yl)acetohydrazide $(1 \mathrm{~m} . \mathrm{mol})$ was dissolved in $\mathrm{POCl}_{3} / \mathrm{SOCl}_{2}(3 \mathrm{~mL})$. The reaction was stirred on magnetic stirrer under reflux for $3 \mathrm{~h}$ and the mixture was cooled to R.T and poured into crushed ice. The solution of $\mathrm{Na}_{2} \mathrm{CO}_{3}(10 \%)$ added to the reaction mixture where a solid mass was separated out. The crude was filtered and washed with water. It was purified by using ethanol to give compound $\mathbf{8}$.

\section{Physical properties and spectral analysis of synthesized 1,3,4-oxadiazoles derivatives}

2-Phenyl-5-((1-phenyl-3,4-dihydroisoqunoline-2(1H)-yl)methyl)-1,3,4-oxadiazole (8a)

${ }^{1} \mathbf{H}$ NMR $(400 \mathrm{MHz}, \mathrm{CDCl} 3) \delta: 8.80(\mathrm{~d}, \mathrm{~J}=8.8 \mathrm{~Hz}, 1 \mathrm{H}), 8.24(\mathrm{~d}, \mathrm{~J}=9.2 \mathrm{~Hz}, 1 \mathrm{H}), 7.43-7.01$ $(\mathrm{m}, 11 \mathrm{Ar}-\mathrm{H}), 6.72(\mathrm{~d}, \mathrm{~J}=8 \mathrm{~Hz}, 1 \mathrm{H}), 4.85(\mathrm{~s}, 1 \mathrm{H}), 3.37-3.23(\mathrm{~m}, 2 \mathrm{H}), 2.97-2.87(\mathrm{~m}, 4 \mathrm{H})$.

${ }^{13} \mathrm{C}$ NMR $\left(100 \mathrm{MHz}, \mathrm{CDCl}_{3}\right) \delta: 163.6,143.1,142.0,135.9,129.3,129.2,128.5,128.3$, 128.2, 128.1, 127.6, 126.6, 126.3, 126.1, 126.0, 58.7, 49.9, 26.7. LCMS $(\mathrm{m} / \mathrm{z}): 366.93$. Molecular formula: $\mathrm{C}_{24} \mathrm{H}_{21} \mathrm{~N}_{3} \mathrm{O}$; Elemental analysis; Calculated: C-78.45, H-5.76, N-11.44 O-4.35; obtained: C-78.46, H-5.75, N-11.42, O-4.34.

4-(5-((1-Phenyl-3,4-dihydro isoquinoline-2-(1H)-yl)methyl))-1,3,4-oxadiazole-2-yl (8b)

${ }^{1} \mathbf{H}$ NMR $\left(400 \mathrm{MHZ}, \mathrm{CDCl}_{3}\right) \delta: 9.01(\mathrm{~s}, 1 \mathrm{H}), 8.30(\mathrm{~d}, \mathrm{j}=8.8 \mathrm{~Hz}, 1 \mathrm{H}), 8.14(\mathrm{~d}, \mathrm{~J}=8.8 \mathrm{~Hz}, 1 \mathrm{H})$, 7.42-7.01 (m, 9H), $6.72(\mathrm{~d}, \mathrm{~J}=8 \mathrm{~Hz}, 1 \mathrm{H}), 4.96(\mathrm{~s}, 1 \mathrm{H}), 3.37-3.32(\mathrm{~m}, 2 \mathrm{H}), 2.72-2.07(\mathrm{~m}, 4 \mathrm{H})$. ${ }^{13} \mathrm{C}$ NMR $\left(100 \mathrm{MHz}, \mathrm{CDCl}_{3}\right) \delta: 16.2 .6,157.6,143.1,142.4,131.9,128.2,127.9,126.6$, 126.3, 126.1, 125.8, 118.6, 116.2, 115.9, 54.4, 50.5, 48.9, 27.5. LCMS $(\mathrm{m} / \mathrm{z}): 384.21$, molecular formula: $\mathrm{C}_{24} \mathrm{H}_{21} \mathrm{~N}_{3} \mathrm{O}_{2}$. Elemental analysis; Calculated: C-75.18.08, H-5.52, N-10.96, O-8.35; Obtained: C-75.20, H-5.49, N-10.95, O-8.33.

2-(3,4-Dimethoxyphenyl)-5-((1-phenyl-3,4-dihydro isoquinoline-2(1H)-yl)methyl)1,3,4-oxadiazole $(8 \mathrm{c})$

${ }^{1} \mathbf{H}$ NMR $\left(400 \mathrm{MHZ}, \mathrm{CDCl}_{3}\right) \delta: 8.10(\mathrm{~d}, \mathrm{~J}=7.6 \mathrm{~Hz}, 1 \mathrm{H}), 7.937 .02(\mathrm{~m}, 10 \mathrm{H}), 6.9(\mathrm{~d}, \mathrm{~J}=7.6$ $\mathrm{Hz}, 1 \mathrm{H}), 4.96(\mathrm{~s}, 1 \mathrm{H}), \quad 3.89(\mathrm{~S}, 9 \mathrm{H}), 3.41-3.30 \quad(\mathrm{M}, 2 \mathrm{H}), 2.79-2.60(\mathrm{~m}, 4 \mathrm{H}) .{ }^{13} \mathbf{C}$ NMR $(100$ $\left.\mathrm{MHz}, \mathrm{CDCl}_{3}\right) \delta: 165.6,148.9,143.3,142.2,135.1,132.6,129.1,128.2,127.8,126.3,125.5$, 125.3, 119.2, 112.6, 110.8, 108.1, 55.3, 50.6, 48.8, 27.5. LCMS $(\mathrm{m} / \mathrm{z}): 417.19$. Molecular formula: $\mathrm{C}_{25} \mathrm{H}_{27} \mathrm{~N}_{3} \mathrm{O}_{3}$. Elemental analysis: Calculated: C-71.92, H-6.52, N-10.06, O-11.50. Obtained: C-71.93, H-6.50, N-10.04, O-11.48.

2-(4-Chlorophenyl)-5-((1-phenyl-3,4-dihydroisoqunoline-2(1H)-yl)methyl)-1,3,4oxadiazole $(\mathbf{8 d})$

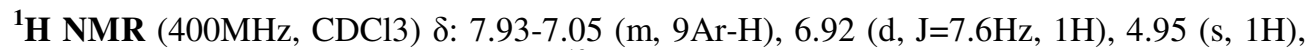
3.41-3.30 (m, 2H), 2.99-2.90 (m, 4H). ${ }^{13}$ CNMR (100 MHz, $\left.\mathrm{CDCl}_{3}\right) \delta: 164.0,143.5,142.5$, 136.6, 133.4, 129.3, 129.2, 128.9, 128.8, 128.6, 128.5, 126.7, 126.3 126.2, 124.9, 59.02, 50.92, 
49.5, 27.09. LCMS $(\mathrm{m} / \mathrm{z})$ : 400.83. Molecular formula: $\mathrm{C}_{24} \mathrm{H}_{20} \mathrm{ClN}_{3} \mathrm{O}$. Elemental analysis; Calculated: C-71.73, H-5.02, Cl-8.82, N-10.46, O-3.98. Obtained: C-71.75, H-5.01, Cl-8.80, N-10.44, O-3.97.

2-(4-Bromophenyl)-5-((1-phenyl-3,4-dihydroisoqunoline-2(1H)-yl)methyl)-1,3,4oxadiazole $(\mathbf{8 e})$

${ }^{1} \mathbf{H}$ NMR (400 MHZ, $\left.\mathrm{CDCl}_{3}\right) \delta: 8.40(\mathrm{~d}, \mathrm{~J}=8.8 \mathrm{~Hz}, 1 \mathrm{H}), 8.24(\mathrm{~d}, \mathrm{~J}=9.2 \mathrm{~Hz}, 1 \mathrm{H}), 7.44-7.01$ $(\mathrm{m}, \operatorname{Ar}-\mathrm{H}), 6.72(\mathrm{~d}, \mathrm{~J}=8 \mathrm{~Hz}, 1 \mathrm{H}), 4.85(\mathrm{~s}, 1 \mathrm{H}), 3.37-3.23(\mathrm{~m}, 2 \mathrm{H}), 2.97-2.07(\mathrm{~m}, 4 \mathrm{H})$. ${ }^{13}$ C NMR $\left(100 \mathrm{MHz}, \mathrm{CDCl}_{3}\right) \delta: 164.1,143.5,142.7,136.9,133.2,129.7,129.3,128.3$, 128.3, 128.2, 126.6, 126.5, 126.2, 125.2, 123.0, 59.7, 50.5, 49.5, 26.7. LCMS $(\mathrm{m} / \mathrm{z}): 447.01$ $(\mathrm{M}+2)$. Molecular formula: $\mathrm{C}_{24} \mathrm{H}_{20} \mathrm{BrN}_{3} \mathrm{O}$. Elemental analysis: Calculated: C-64.58, H-4.52, Br-17.90, N-9.41, O-3.58. Obtained: C-64.59, H-4.50, Br-17.89, N-9.40, O-3.50.

2-(4-Nitrophenyl)-5-((1-phenyl-3,4-dihydroisoqunoline-2(1H)-yl)methyl)-1,3,4oxadiazole $(8 \mathrm{f})$

${ }^{1} \mathbf{H}$ NMR $\left(400 \mathrm{MHZ}, \mathrm{CDCl}_{3}\right) \delta: 8.13(\mathrm{~d}, \mathrm{~J}=8 \mathrm{~Hz}, 1 \mathrm{H}), 7.62-7.02(\mathrm{~m}, \mathrm{Ar}-\mathrm{H}), 6.73(\mathrm{~d}, \mathrm{~J}=8 \mathrm{~Hz}$, $1 \mathrm{H}), 4.91(\mathrm{~s}, 1 \mathrm{H}), 3.38-3.23(\mathrm{~m}, 2 \mathrm{H}), 2.97-2.86(\mathrm{~m}, 4 \mathrm{H}) .{ }^{13} \mathbf{C ~ N M R}\left(100 \mathrm{MHz}, \mathrm{CDCl}_{3}\right) \delta$ : 164.6, 147.9, 143.4, 142.8, 136.1, 132.0, 129.1, 128.7, 128.6, 128.5, 128.5, 126.6, 126.3, 126.1, 121.8, 58.3, 50.9, 49.5, 28.49. LCMS (m/z): 413.10. Molecular formula: $\mathrm{C}_{24} \mathrm{H}_{20} \mathrm{~N}_{4} \mathrm{O}_{3}$. Elemental analysis; Calculated: C-69.89, H-4.89, N-13.58, O-11.64. Obtained: C-69.90, H4.87, N-13.57, O-11.63.

4-(5-((1-Phenyl-3,4-dihydroisoquinoline-2(1H)-yl)methyl)-1,3,4-oxadiazole-2yl) benzonitrile $(8 \mathrm{~g})$

${ }^{1} \mathbf{H}$ NMR $\left(400 \mathrm{MHZ}, \mathrm{CDCl}_{3}\right) \delta: 8.08(\mathrm{~d}, \mathrm{~J}=7.61 \mathrm{~Hz}, 1 \mathrm{H}), 7.93-7.01(\mathrm{~m}, 10 \mathrm{Ar}-\mathrm{H}), 6.76(\mathrm{~d}$, $\mathrm{J}=7.6 \mathrm{~Hz}, 1 \mathrm{H}), 5.06(\mathrm{~s}, 1 \mathrm{H}), 3.72-3.30(\mathrm{~m}, 2 \mathrm{H}), 2.89-2.60(\mathrm{~m}, 4 \mathrm{H}) .{ }^{13} \mathbf{C}$ NMR $(100 \mathrm{MHz}$, $\left.\mathrm{CDCl}_{3}\right) \delta: 163.9,159.8,143.2,141.6,132.9,131.8,130.2,129.0,128.2,128.1,128.0,126.3$, 126.1, 126.0, 125.6, 118.6, 112.1, 55.5, 50.5, 49.5, 26.9. LCMS $(\mathrm{m} / \mathrm{z}): 392.68$, molecular formula: $\mathrm{C}_{25} \mathrm{H}_{20} \mathrm{~N} 4 \mathrm{O}$. Elemental analysis; Calculated: C-76.12, H-5.62, N-14.20, O-4.06, obtained: C-76.14, H-5.60, N-14.19, O-4.04.

2-(3-Bromopyridine-4-yl)-5-((1-phenyl-3,4-dihydroisoqunoline-2(1H)-yl)methyl)1,3,4-oxadiazole $(\mathbf{8 h})$

${ }^{1} \mathbf{H}$ NMR $\left(400 \mathrm{MHZ}, \mathrm{CDCl}_{3}\right) \delta: 8.70(\mathrm{~d}, \mathrm{~J}=8.8 \mathrm{~Hz}, 1 \mathrm{H}), 8.54(\mathrm{~d}, \mathrm{~J}=8.8 \mathrm{~Hz}, 1 \mathrm{H}), 7.43-7.02(\mathrm{~m}$, 9H), $6.62(\mathrm{~d}, \mathrm{~J}=8.4 \mathrm{~Hz}, 1 \mathrm{H}), 4.95(\mathrm{~s}, 1 \mathrm{H}), 3.67-3.23(\mathrm{~m}, 2 \mathrm{H}), 2.97-2.57(\mathrm{~m}, 4 \mathrm{H}) .{ }^{13} \mathbf{C} \mathbf{~ N M R}$ $\left(100 \mathrm{MHz}, \mathrm{CDCl}_{3}\right) \delta: 164.6,148.8,148.3,146.8,143.2,142.1,136.2,129.1,128.4,128.3$, 128.1, 126.6, 126.3, 126.1, 120.83, 119.4, 59.4, 50.9, 27.5. LCMS $(\mathrm{m} / z): 447.11(\mathrm{M}+1)$. Molecular formula: $\mathrm{C}_{23} \mathrm{H}_{19} \mathrm{~N}_{4} \mathrm{O}$. Elemental analysis; Calculated: C-61.75, H-4.28, Br-17.86, N-12.52, O-3.58. Obtained: C-61.77, H-4.26, Br-17.85, N-12.50, O-3.55.

Bioevalution of the new synthesized molecule

The new synthesized target $(\mathbf{8 a - 8 h})$ molecules were studied for their biological activity at microbiology laboratory, in Department of Organic Chemistry, Andhra University. Eight target molecules were examined for the anti bacterial activity (i.e., gram negative, gram positive) and fungal activity using Petri disc method. Among the eight new molecules, only two molecules $(\mathbf{8 a}, \mathbf{8 h})$ didn't show the anti bacterial activity. Compounds $(\mathbf{8 b}, \mathbf{8 c}, \mathbf{8 d}, \mathbf{8 e})$ showed high activity. Compounds $\mathbf{8 f} \& \mathbf{8 g}$ showed less activity. The activity results in terms of zones of inhibition values were presented in Table 1 . 
Table 1. Zone of inhibition antibacterial activity of compounds

\begin{tabular}{cccccccc}
\hline S.No & $\begin{array}{c}\text { Compound } \\
\text { Name }\end{array}$ & \multicolumn{2}{c}{$\begin{array}{c}\text { Gram negative } \\
\text { (Escherichia cole) }\end{array}$} & \multicolumn{2}{c}{$\begin{array}{c}\text { Gram positive } \\
\text { (Bacillius subtilis) }\end{array}$} & \multicolumn{2}{c}{$\begin{array}{c}\text { Fungi } \\
\text { (Aspergillus niger) }\end{array}$} \\
\hline & & $10 \mathrm{mg}$ & $5 \mathrm{mg}$ & $10 \mathrm{mg}$ & $5 \mathrm{mg}$ & $10 \mathrm{mg}$ & $5 \mathrm{mg}$ \\
\hline 1 & $\mathbf{8 a}$ & 0 & 0 & 0 & 0 & 0 & 0 \\
2 & $\mathbf{8 b}$ & 13 & 11 & 15 & 10 & 13 & 8 \\
3 & $\mathbf{8 c}$ & 8 & 7 & 18 & 14 & 15 & 8 \\
4 & $\mathbf{8 d}$ & 17 & 14 & 18 & 11 & 18 & 13 \\
5 & $\mathbf{8 e}$ & 15 & 7 & 12 & 6 & 10 & 7 \\
6 & $\mathbf{8 f}$ & 7 & 7 & 12 & 8 & 8 & 0 \\
7 & $\mathbf{8 g}$ & 0 & 0 & 13 & 8 & 15 & 7 \\
8 & $\mathbf{8 h}$ & 0 & 0 & 0 & 0 & 0 & 0 \\
\hline
\end{tabular}

\section{Results and Discussion}

\section{Chemistry}

The target molecules (8a-8h) were synthesized and characterized by advanced spectral techniques $\left({ }^{1} \mathrm{H}\right.$ NMR, ${ }^{13} \mathrm{C}$ NMR \& Mass) for the first time and reported. We further studied anti bacterial activity and anti fungal activity of the above synthesized derivatives. Initially, the direct condensation of 2-phenylethyl amine $\mathbf{1}$ and benzoic acid $\mathbf{2}$ was performed which leads to the $N$-(2-phenylethyl)benzamide $\mathbf{3}$ in excellent yield. Then, the obtained compound $\mathbf{3}$ was cyclised by Bischler-Napierlaski method to yield 1-phenyl, 3,4-dihydroisoquinoline (imine) 4. Reduction of imine $\mathbf{4}$ resulted in racemic 1-Phenyl-1,2,3,4-Tetrahydroisoquinoline $\mathbf{5}$. Compound $\mathbf{5}$ is treated with ethyl 2-bromoacetate to obtain ester derivative 6. Ester $\mathbf{6}$ on treatment with hydrazine hydrocholoride furnished hydrazide derivative $\mathbf{7}$ from which target oxadiazoles 8 were prepared upon reaction with benzoic acids.

During the synthesis of oxadiazoles, two cyclizing reagents were used $\mathrm{SOCl}_{2} / \mathrm{POCl}_{3}$. In these two reagents, we used former reagent which gave better yields, low reaction time, and minimize the formation of biproducts than the lateral reagent. We used several substituted benzoic acids to check the scope of the reaction. Benzoic acids having both electron withdrawing groups such as $\mathrm{CN}, \mathrm{NO}_{2}$ and electron donating groups such as $\mathrm{OMe}, \mathrm{OH}$ participated in the reaction. Besides, we observed electron releasing group $\left(-\mathrm{OCH}_{3},-\mathrm{OH}\right)$ of aryl acids enhanced the rate of reaction than the electron withdrawing group $\left(-\mathrm{CN},-\mathrm{NO}_{2}\right)$ of aryl acids. All the reactions performed went smoothly under the reaction conditions to furnish excellent yields $(>90 \%)$ in the time span of 3 to 4 hours.

\section{Biology}

The anti bacterial activity results indicated that presence of electron releasing group $\left(-\mathrm{OCH}_{3}\right.$, $-\mathrm{OH})$ at para position of the phenyl ring exhibit excellent biological activity than the electron withdrawing group $\left(-\mathrm{CN},-\mathrm{NO}_{2}\right)$. On the other hand, from the basis of biological active data, it is clear that biological activity was enhanced with the presence of halogen $(\mathrm{Cl}, \mathrm{Br})$ groups on aryl group coming from benzoic acid

\section{Conclusion}

Herein, we reported for the first time 1,3,4-oxadiazole derivatives (8a-8h) from tetrahydroisoquinoline using cyclisation reagent.Among all the synthesized derivatives were observed. 8b, 8c compound showed good yield in less reaction time. Eight new target molecules were examined for the antibacterial activity (i.e., gram negative, gram positive) 
and fungal activity. The new synthesized target $(\mathbf{8 a - 8 h})$ molecules were studied for the biological activity at Microbiology laboratory, in Dept of Organic Chemistry, Andhra University. Among the eight target new molecules, only two molecules $(\mathbf{8 a}, \mathbf{8 h})$ did not show the antibacterial activity. The biological active analysis data for $\mathbf{8 b}, \mathbf{8 c}, \mathbf{8 d}$ compounds were found to exhibit good activity among all the derivatives.

\section{Acknowledgement}

The auther is highly greatful to Head, department of organic Chemistry, AU for sanction permission to carry out these investigations for my $\mathrm{Ph}$. D thesis research work. We are grateful to Andhra University, for providing NMR spectra data (NMR research center).

\section{References}

1. Wei Y, Dang L, Zhang X, Cui W and Wei H, Flu Pha Equ., 2012, 318, 13-18.

2. Demadrille R, Firon M, Leroy J, Rannou P and Pron A, Adv Funct Mater., 2005, 15(9), 1547-1552; DOI:10.1002/adfm.200500117

3. Jumat S, Nadia S, Ayad H and Hiba I E Y, J Appl Sci Res., 2010, 6(7), 866-870.

4. Sowjanya C, Rama Bharathi V, Devi G and Rajitha G, J Chem Pharm Res., 2011, 3(6), 212-216.

5. Maslat A O, Abussaud M, Tashtoush H and Al-Talib M, Pol J Pharmacol., 2002, 54(1), 55-59.

6. Bhatia S and Gupta M, J Chem Pharm Res., 2011, 3(3), 137-147.

7. Patel K, Chamndran J E, Shah R, Vijaya J and Sreenivasa G M, Int J Pharm Bio Sci., 2010, 1(3), 1-13.

8. Pattan S R, Rabara P A, Pattan J S, Bukitagar A A and Wakale V S, Indian J Chem., 2009, 48B, 1453-1456.

9. Kucukguzel S G, Kucukguzel I, Tatar E, Rollas S, Sahin F, Gulluce M, De Clercq E and Kabasakal L, Eur J Med Chem., 2007, 42(7), 893-901; DOI:10.1016/j.ejmech.2006.12.038

10. Holla B S, Poojary K N, Bhat K S, Ashok M and Poojary B, Indian J Chem., 2005, 44B, 1669-1673.

11. El-Sayeda W A, El-Essawyb F A, Alib O M, Nasr B S, Abdalla M M and Abdel Rahman A A, Z Naturforsch., 2009, 64C, 773-778.

12. Cena C, Bertinaria M, Boschi D, Giorgis M and Gasco A, ARKIVOC., 2006, 7, 301-309.

13. Husain A and Ajmal M, Acta Pharm., 2009, 59(2), 223-233.

14. Amir $\mathrm{M}$ and Shikha K, Eur J Med Chem., 2004, 39(6), 535-545; DOI:10.1016/j.ejmech.2004.02.008

15. Zou X J, Lai L H, Jin G Y and Zhang Z X, J Agric Food Chem., 2002, 50(13), 3757 3760; DOI:10.1021/jf0201677

16. Mohan T P, Vishalakshi B, Bhat K S, Rao K S and Kendappa G N, Indian J Chem., 2004, 43B, 1798-1801.

17. Dewangan D, Pandey A, Sivakumar T, Rajavel R and Dubey R D, Int J Chem Tech Res., 2010, 2(3), 1397-1412.

18. Zarghi A, Hamedi S, Tootooni F, Amini B, Sharifi B, Faizi M, Tabatabai S A and Shafiee A, Sci Pharm., 2008, 76(2), 185-201; DOI:10.3797/scipharm.0803-10

19. Zarghi A, Tabataba A A, Faizi M, Ahadian A, Navabi P, Zanganeh V and Shafiee A Q, Bioorg Med Chem Lett., 2005, 15(7), 1863-1865.

20. Mogilaiah K and Reddy I N V, Indian J Chem., 2003, 42B, 2124-2125. 